\title{
Incidence, Risk Factors, and Attributable Mortality of Catheter-Related Bloodstream Infections in the Intensive Care Unit After Suspected Catheters Infection: A Retrospective 10-year Cohort Study
}

\author{
Yiyue Zhong · Limin Zhou • Xiaolei Liu · Liehua Deng • \\ Ruona Wu ' Zhengyuan Xia · Guixi Mo · Liangqing Zhang • \\ Zhifeng Liu · Jing Tang (D)
}

Received: January 28, 2021 / Accepted: February 27, 2021 / Published online: April 16, 2021

(c) The Author(s) 2021

\begin{abstract}
Introduction: Catheter management strategies for suspected catheter-related bloodstream infection (CRBSI) remain a major challenge in intensive care units (ICUs). The objective of this study was to determine the incidence, risk factors, and mortality attributable to CRBSIs in those patients.
\end{abstract}

Methods: A population-based surveillance on suspected CRBSI was conducted from 2009 to 2018 in a tertiary care hospital in China. We used the results of catheter tip culture to

Supplementary Information The online version contains supplementary material available at https:// doi.org/10.1007/s40121-021-00429-3.

Y. Zhong · L. Zhou - X. Liu · R. Wu · Z. Xia .

G. Mo · L. Zhang · J. Tang ( $\varangle)$

Department of Anesthesia, Affiliated Hospital of

Guangdong Medical University, Zhanjiang,

Guangdong, China

e-mail: tanglitangjing@126.com

L. Deng

Department of Critical Care Medicine, Affiliated

Hospital of Guangdong Medical University,

Zhanjiang, Guangdong, China

e-mail: glinson@126.com

\section{Z. Liu}

Department of Critical Care Medicine, General

Hospital of Southern Theatre Command of PLA, Guangzhou, China

e-mail: zhifengliu7797@163.com identify patients with suspected CRBSIs. Demographics, systemic inflammatory response syndrome (SIRS) criteria, interventions, and microorganism culture results were analysed and compared between patients with and without confirmed CRBSIs. Univariate and multivariate analyses identified the risk factors for CRBSIs, and attributable mortality was evaluated with a time-varying Cox proportional hazard model.

Results: In total, 686 patients with 795 episodes of suspected CRBSIs were included; $19.2 \%$ (153/795) episodes were confirmed as CRBSIs, and $17.4 \%(119 / 686)$ patients died within 30 days. The multifactor model shows that CRBSIs were associated with fever, hypotension, acute respiratory distress syndrome, hyperglycaemia and the use of continuous renal replacement therapy. The AUC was $77.0 \%$ (95\% CI $73.3 \%-80.7 \%)$. The population attributable mortality fraction of CRBSI in patients was $18.2 \%$, and mortality rate did not differ significantly between patients with and without CRBSIs (95\% CI 0.464-1.279, $P=0.312$ ).

Conclusions: This initial model based on the SIRS criteria is relatively better at identifying patients with CRBSI but only in domains of the sensitivity. There were no significant differences in attributable mortality due to CRBSI and other causes in patients with suspected CRBSI, which prompt catheter removal and re-insertion of 
new catheter may not benefit patients with suspected CRBSIs.

Trial Registration: China Clinical Trials Registration number; ChiCTR1900022175.

Keywords: Catheter-related bloodstream infections; Central venous catheter; Intensive care units; Mortality; Risk factor; Systemic inflammatory response syndrome

\section{Key Summary Points}

\section{Why carry out this study?}

Catheter management strategies for suspected catheter-related bloodstream infection (CRBSI) remain a major challenge.

There is an urgent need to develop strong practical evidence to identify CRBSI for prevent unnecessary catheter removal and subsequent harm to patients.

To knowledge the attributable mortality of CRBSI is important that a key factor to the decisions made regarding the suspected CRBSIs management strategy in patients, but it is remains uncertain.

\section{Why carry out this study?}

This initial model based on the SIRS criteria is relatively better at identifying patients with CRBSI but only in domains of the sensitivity.

There were no significant differences in attributable mortality due to CRBSI and other causes in patients with suspected CRBSI, which prompt catheter removal and re-insertion of new catheter may not benefit patients with suspected CRBSIs.

\section{DIGITAL FEAUTRES}

This article is published with digital features, including a summary slide, to facilitate understanding of the article. To view digital features for this article go to https://doi.org/10. 6084/m9.figshare.14125796.

\section{INTRODUCTION}

Catheter-related bloodstream infections (CRBSIs) are common intensive care unit (ICU)-acquired infections [1,2], which have a significant effect on morbidity, mortality and associated health care costs [3-5]. CRBSIs are an important type of infection in ICU patients with sepsis [2], which is defined as life-threatening organ dysfunction caused by a dysregulated host response to infection [6], which compromises host homeostasis by the induction of the iron-sequestering ferritin $\mathrm{H}$ chain in response to polymicrobial infections and causes systemic inflammatory response syndrome (SIRS) $[7,8]$. However, to date, there is a lack of specific and reliable data to show that the indicators that are sensitive for the diagnosis of suspected sepsis can be used to identify CRBSI due to suspected but unconfirmed CRBSI. In addition, the attributable mortality to CRBSI remains uncertain and ranges from $-12.24 \%$ to $25.96 \%$ [9]; the mortality is an important factor affecting the decisions made regarding the central venous catheters (CVCs) management strategy in patients with suspected CRBSIs [2,9-11]. A recent systematic review reported that there was no robust evidence to guide the selection of the management strategy for patients with suspected CRBSIs [1], and knowledge of the attributable mortality of CRBSI is important.

The primary objective of this study was to determine the incidence of, risk factors for, and mortality attributable to CRBSIs in ICU patients with suspected but unconfirmed CRBSIs. Additionally, we also assessed the incidence of and mortality attributable to other infections during the same episodes.

\section{METHODS}

\section{Study Design and Participants}

This was a retrospective cohort study conducted at the Affiliated Hospital of Guangdong Medical 
University, Zhanjiang, Guangdong, China. The ethics committee of institution approved this study and waived the need for informed consent on March 13, 2019 (PJ2018-066). This study was registered with the China Clinical Trials Registry on March 28, 2019 (ChiCTR1900022175). From January 2009 to December 2018. Antimicrobial catheters were not used in this ICU. According to the clinical practice guidelines of the Infectious Diseases Society of America (IDSA), catheter cultures were performed when a catheter was removed due to a suspected CRBSI. [12] We analyzed all data from patients aged $\geq 18$ years who had suspected CRBSIs who had cultures performed of their catheter tips. The following exclusion criteria were used: malignant blood disease, chemotherapy or radiotherapy in the same period, length of stay less than $48 \mathrm{~h}$, serious sepsis diagnosed on admission, CRBSI diagnosed of the old catheter after the reinsertion of new catheter, implantation of intravascular foreign bodies within the past month, and human immunodeficiency virus (Fig. 1).

\section{Variables and Data Sources}

We used the medical charts and laboratory electronic database to collect demographic data; diagnoses; comorbidities; disease status on ICU admission; CVC information, treatment interventions; clinical symptoms; and laboratory values at the onset of the suspected CRBSI; the results of microbial cultures within $24 \mathrm{~h}$ before catheter removal; and 30-day mortality. Two classification systems were used to assess patients with times between ICU admission and the development of a suspected CRBSI: The Acute Physiology and Chronic Health Evaluation (APACHE) II score and Sequential Organ Failure Assessment (SOFA) score [2,6].

To assess the possible risk factors, we used the transformed data that were stratified according to the SIRS criteria of International Sepsis Definitions Conference (ISDC), including five aspects variable: general variable, inflammatory variables, hemodynamic variables, organ dysfunction variables, tissue perfusion variables. [13] However, for plasma glucose levels, we used the highest mean level three days before CVC removal, and the transformed data were stratified according to the values recommended in the international guidelines, with a plasma glucose target $\leq 10 \mathrm{mmol} / \mathrm{L}$ [14].

The outcome of this study was 30-day mortality in patients with suspected CRBSIs, and the mortality after 30 days was considered to be less likely to be related to CRBSIs $[11,12]$.

\section{Definitions}

Catheter tip colonization was defined as a catheter tip with 15 or more colony-forming units [11]. The CRBSI definition adhered to the IDSA guidelines [12], which required the catheter tip to be colonized by a microorganism that was phenotypically the same as a microorganism isolated from a peripheral blood culture. A suspected CRBSI was identified when a patient developed a new episode of fever or sepsis [11], with at least 1 additional parameter described in the 2001 ISDC guidelines noted in the progress notes in the medical charts [13]. Fever was defined as a temperature $>38.3^{\circ} \mathrm{C}[13]$. Sepsis was defined as lifethreatening organ dysfunction caused by a dysregulated host response to infection, as in the Third International Consensus Definitions [6].

\section{Study Sample Size}

The study sample included patients aged $\geq 18$ year who were suspected CRBSI from January 2009 to December 2018. All available patients were included: 642 in the non-CRBSI group and 153 in the CRBSI group. Assuming a mortality rate of $25.96 \%$ with CRBSI [15] and $10.9 \%$ with ICU-acquired infections [2], we calculated that group sample sizes of 209 in non-CRBSI group and 105 in CRBSI group achieve $80 \%$ power to detect a ratio in the group proportions of 0.5 by the PASS 11 software (NCSS, LLC. Kaysville, Utah, USA. www.ncss. com.). 


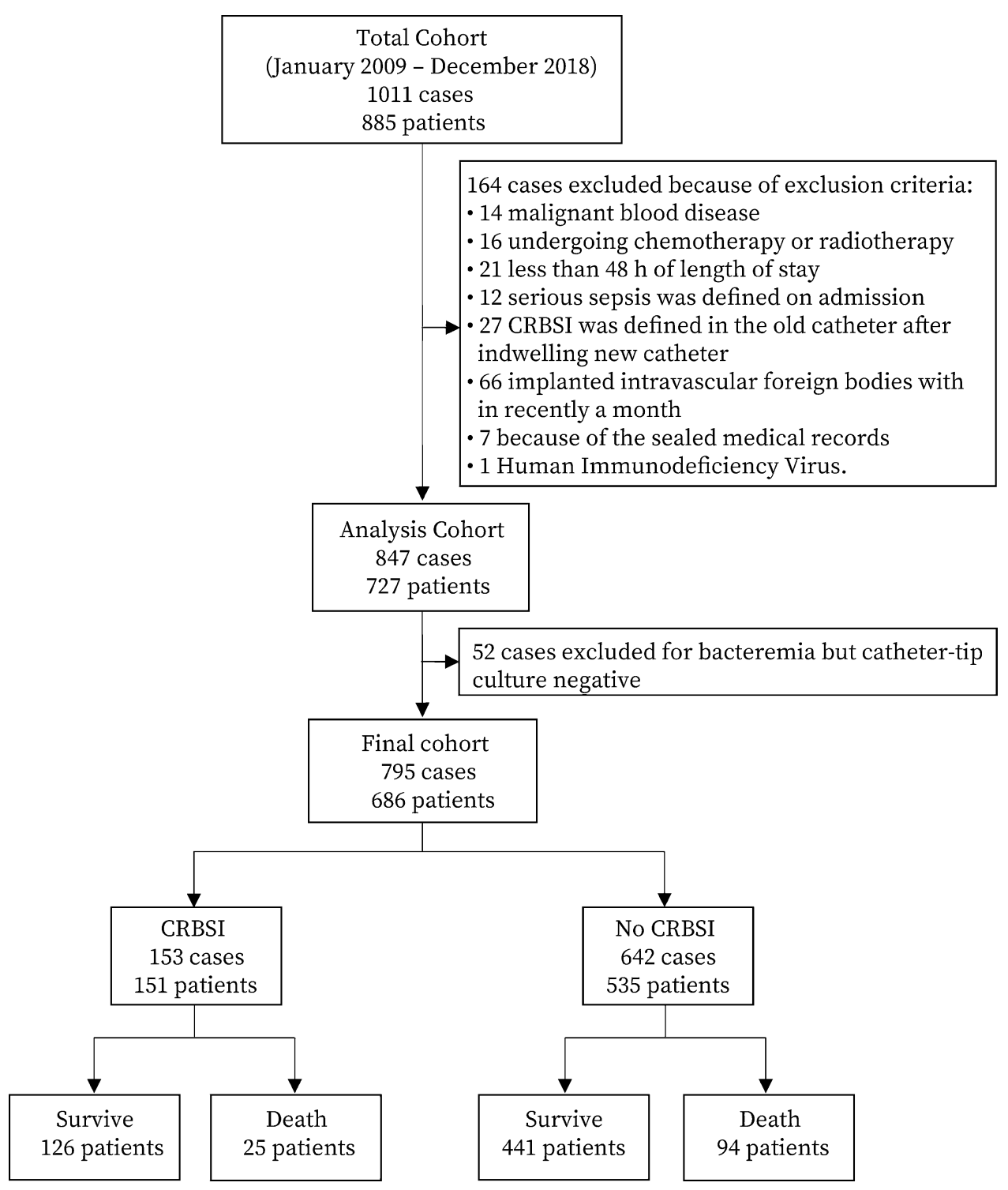

Fig. 1 Flow diagram and control patients for analysis

\section{Statistical Analysis}

Categorical variables are shown as numbers (\%), and continuous parameters are shown as the means (SDs) or medians (IQRs). Comparisons were made with Fisher's exact test for categorical data and the Wilcoxon test for continuous data. In the final cohort, 52 cases of bacteraemia were excluded based on the absence of catheter colonization; these cases were considered possible deviations from the diagnosis of bacteraemia based on the analysis of the clinical symptoms. For the survival analysis, all patients were analyzed in aggregate and stratified by survival status at 30 days, and the last data were used as covariates when one patient had two or more episodes of suspected CRBSIs.

The hypothesis of this study was that the attributable mortality of CRBSI might be higher than other infections. The statistical analysis was performed in three steps. First, we used univariate and multivariate analyses to determine the risk factors for CRBSI. The quartiles of APACHE II scores and of SOFA scores per 
specific patient population were used as covariables $[2,6]$. A multivariable generalized linear model was used to evaluate the risk factors for CRBSI. A nonparametric receiver operating characteristic curve was constructed, and the area under the curve (AUC) was calculated. In the second step, we evaluated the mortality associated with CRBSI in the entire cohort using a time-varying Cox proportional hazard model [16], and the fraction of attributable mortality was used after the final model estimation command, the parameters of which were interpreted as log rate ratios [17]. Third, we assessed the incidence of and mortality attributable to other infections in patients with suspected CRBSIs during the same period. All statistical tests were two-tailed, and significance was set at $\alpha=0.05$.

The statistical analyses were performed with Stata/SE 15.1 (Stata Corp LLC 4905 Lakeway Drive College Station, TX 77,845 USA).

\section{RESULTS}

\section{Baseline Characteristics}

A total of 7653 critically ill patient with 12,245 CVCs were admitted to the ICU, among which CVC removal was undergone in 5623 (45.9\%) cases for various reasons. Among 1011 (18.0\%) cases whose CVCs were removed due to suspected CRBSI, 164 cases were excluded because they met the exclusion criteria. We studied 686 patients with 795 ICU episodes in the final analysis (Fig. 1); 93 patients had at least 2 or more episodes of suspected CRBSIs. Of all episodes of suspected CRBSIs, 153 (19.2\%) were defined as CRBSIs based on microorganism culture results from among 627 (78.9\%) documented infections. Figure 2 shows that the range of suspected CRBSIs has expanded, but the rate of confirmed CRBSIs has trended downward, especially in 2018, in which only $8.3 \%$ of suspected CRBSIs were confirmed. In addition, regarding the pathogenic microorganisms causing CRBSIs, we found 40 (25.5\%) gram-positive cocci, 79 (50.3\%) gram-negative bacilli and 38 (24.2\%) fungi; 4 of the 153 cases of CRBSIs had multiple microbes colonizing the same catheter tip (Supplemental e-Table 1).

\section{Risk Factors Analysis}

A total of 795 ICU episodes were included in the analysis of the risk factors for CRBSI in patients with suspected CRBSIs. We did not find significant differences between the CRBSI and nonCRBSI groups in the baseline cohort with regard to age, sex, or admission diagnosis (Supplemental e-Table 2). We used a multifactor generalized linear model to assess the risk factors for CRBSI based on the results of univariate analysis (Table 1 ). The model showed an elevated risk of CRBSI in patients with fever $\left(>38.3^{\circ} \mathrm{C}\right.$ ), arterial hypotension (mean arterial pressure $[\mathrm{MAP}]<70 \mathrm{mmHg}$ ), hyperglycemia (plasma glucose $>10 \mathrm{mmol} / \mathrm{L}$ ), acute respiratory distress syndrome (ARDS), and the use of continuous renal replacement therapy (CRRT). However, a lower risk of CRBSI was observed in patients with diabetes mellitus (95\% CI $0.194-0.644, \quad P=0.001) \quad$ and creatinine $\geq 133 \mathrm{mmol} / \mathrm{L} \quad(95 \% \quad$ CI $\quad 0.173-0.538$, $P<0.001)$. The AUC of the model was $77.0 \%$ (95\% CI 73.3\%-80.7\%). In the expanded baseline cohort, patients in whom CVCs were reinserted (within $24 \mathrm{~h}$ ) had a higher relative risk of CRBSIs than those in whom CVCs were not reinserted $(30.0 \%$ vs $19.2 \%$, 95\% CI 1.145-2.121, $\quad P=0.007$ ) (Supplemental e-Table 2). Hence, it may be necessary to perform a more comprehensive assessment of the clinical significance of the relative risk in patients in whom CVCs are reinserted.

\section{Mortality Risk Analysis}

We included all 686 patients in the analysis of survival. The mean age was 62.9 years (SD 17.1), $503(73.3 \%)$ were male, and $183(26.7 \%)$ were female. A total of 119 (17.4\%) patients with suspected CRBSIs died within 30 days. Table 2 showed univariate relative risk factors that there were significant differences between death and survivors.

A multivariate Cox proportional hazards model was used to identify the association of CRBSI with mortality. The models were adjusted for the variables with significant results in univariate analysis. A crude univariate model 


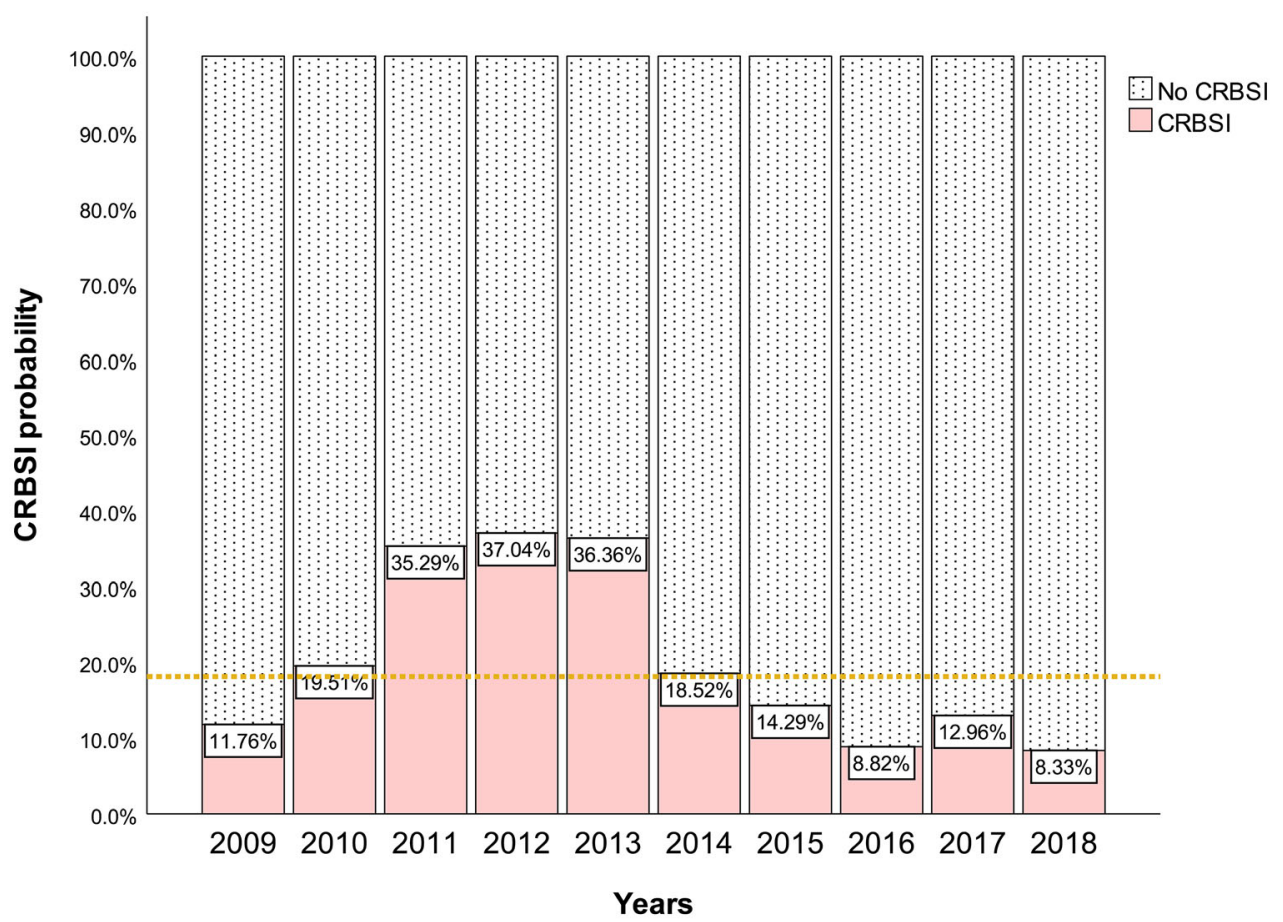

Fig. 2 The incidence of CRBSI in patients with suspected CRBSI from 2009 to 2018. The percentage expression of the documented CRBSI cases. The horizontal dashed line

showed that CRBSI did not increase the hazard ratio for 30-day mortality (95\% CI 0.599-1.433, $P=0.731$ ). Four Cox models were constructed, all of which indicated that CRBSI was not associated with an increase in 30-day mortality (Supplemental e-Table 3). The final model (Table 3) showed that the following four factors were associated with an increased risk of mortality: age ( $>77$ years) (95\% CI 1.087-3.437, $P=0.025)$, APACHE II score $(>25)(95 \%$ CI $1.165-3.938, \quad P=0.014)$, SOFA score $(>13)$ (95\% CI 1.232-3.988, $P=0.008$ ), and the reinsertion of new CVC (95\% CI 1.334-4.066, $P=0.003)$. In contrast, the surgical admission of critically ill patients was associated with lower hazard ratios for 30-day mortality than was the medical admission of critically ill patients (95\% CI 0.227-0.698, $P=0.001$ ).

With regard to the final model, which contains variables that did and did not satisfy the assumption, in the Kaplan-Meier survival analysis (Fig. 3), we used a stratified Cox model (CRBSI and no CRBSI) [17]. We did not find significant differences between the CRBSI group shows the average documented rate of CRBSI in ten years. CRBSI: Catheter-Related Bloodstream Infection

and the no CRBSI group. The test of the proportional hazards assumption for the Cox model [18] showed that it was met (chisquare $=28.20, P=0.1346$ ) but did not meet proportional hazards assumptions in the subgroup of patients with CRBSI who survived (Spearman $=0.142, \quad$ chi-square $=4.33$, $P=0.0375)$.

\section{Population Attributable Mortality Fraction Analysis}

The population attributable fraction analysis was based on the Cox proportional hazards model, and it demonstrated that the fraction of 30-day mortality attributable to CRBSIs was $18.2 \%$ (95\% CI $-21.3 \%$ to $44.8 \%, P=0.318$ ), that attributable to pneumonia was $1.8 \%(95 \%$ CI $-14.5 \%$ to $15.8 \%, P=0.816$ ), and that attributable to multiple infections was $13.8 \%$ (95\% CI $-27.4 \%$ to $41.7 \%, P=0.457$ ) (Supplemental e-Table 4 ). 
Table 1 Generalized linear models for risk factors for CRBSI in patients due to suspected CRBSI

Robust Std. Err $z$-Value RR (95\% Conf. Interval) $P$-value

Admission

APACHE1st quartile (2-14) (reference)

1

APACHE2nd quartile (15-18)

APACHE3rd quartile (19-24)

APACHE4th quartile (25-46)

Comorbidities

Acute Respiratory Distress Syndrome

0.684

Diabetes mellitus

0.108

Kidney injury

0.209

SOFA1st quartile (1-7) (reference)

SOFA2nd quartile (8-9)

SOFA3rd quartile (10-12)

SOFA4th quartile (13-24)

Central venous catheter

Jugular (reference)

Subclavian

0.053

Femoral

0.189

Catheter days $(>7)$

0.322

0.375

0.549

0.176

Vasoconstrictor Agents

Inflammatory variables

Leukocytes (WBC count $>12.000 \mu \mathrm{L}^{-1}$ )

0.204

Leukopenia (WBC count $<4000 \mu \mathrm{L}^{-1}$ )

3.681

Plasma Procalcitonin $(>1 \mathrm{ng} / \mathrm{ml})$

0.235

Organ dysfunction variables

Creatinine increase $(>133 \mu \mathrm{mol} / \mathrm{L})$

0.088

Continuous renal replacement therapy

1.098

Hyperglycemia (plasma glucose $>10 \mathrm{mmol} / \mathrm{L}$ )

0.390

0.480

Glasgow Coma Scale $(<12)$
Severity of disease during suspected CRBSI

$$
-0.07
$$

1

1

$0.8 \quad 1.285(0.696-2.371)$

0.940

$1.71 \quad 1.714(0.924-3.182)$

0.423

0.088

$2.52 \quad 2.194(1.191-4.042)$

0.012

0.001

0.082

- $1.74 \quad 0.421(0.159-1.114)$

1

0.118

0.096

0.197

$-1.290 .628(0.310-1.273)$

1

0.000

$-5.650 .167(0.090-0.311)$

0.218

- $1.23 \quad 0.725(0.434-1.209)$

0.064

$1.85 \quad 1.491(0.977-2.275)$

$2.19 \quad 1.646(1.053-2.573)$

0.029

0.010

0.062

- $1.87 \quad 0.553(0.297-1.030)$

0.740

- $0.33 \quad 0.930(0.605-1.429)$

0.378

$0.88 \quad 2.977(0.264-33.596)$

0.829

$0.22 \quad 1.050(0.676-1.629)$

0.000

- $4.110 .305(0.173-0.538)$

0.000

$4.54 \quad 3.761(2.122-6.666)$

0.014

$2.46 \quad 1.737(1.119-2.697)$

0.078 
Table 1 continued

\begin{tabular}{lllll}
\hline & Robust Std. Err & $\boldsymbol{z}$-Value & RR (95\% Conf. Interval) & $\boldsymbol{P}$-value \\
\hline Activated Partial Thromboplastin Time $(>60 \mathrm{secs})$ & 0.384 & 0.17 & $1.064(0.525-2.158)$ & 0.863 \\
International Normalized Ratio $(>1.5)$ & 0.237 & -0.78 & $0.792(0.440-1.425)$ & 0.436 \\
Thrombocytopenia (platelet count $\left.<100,000 \mu \mathrm{L}^{-1}\right)$ & 0.238 & -0.49 & $0.875(0.513-1.492)$ & 0.623 \\
Tissue perfusion variables & & & & 0.169 \\
Hyperlactatemia (Lactic acid $>1 \mathrm{mmol} / \mathrm{L})$ & 0.175 & -1.37 & $0.713(0.440-1.155)$ & \\
\hline
\end{tabular}

Nonparametric ROC estimation to models: AUC 77.0\% (95\% CI 73.3\%-80.7\%)

APACHE Acute Physiology and Chronic Health Evaluation, CI confidence interval, CRBSI Catheter-related bloodstream infections, $M A P$ mean arterial pressure, $R R$ relative risk, SOFA Sequential Organ Failure Assessment, $W B C$ white blood cell

\section{DISCUSSION}

This large cohort study supports the use of the adjusted SIRS criteria to identify CRBSIs in patients with suspected CRBSIs. There were no significant differences in mortality between patients confirmed with and without CRBSIs who underwent CVC removal due to suspected CRBSIs, regardless of the variables used to adjust the Cox model. However, we determined that the reinsertion of new CVCs was associated with an increased hazard ratio of mortality.

There are five aspects of the SIRS criteria. First, the general variable fever was confirmed to be a specific and sensitive indicator of CRBSIs in this study. The guidelines report that fever is the most sensitive clinical finding that is indicative of CRBSI but that it has poor specificity [12]. In addition, our data did show a relatively higher risk of CRBSIs in patients using the cutoff value for the glucose level of $>10 \mathrm{mmol} / \mathrm{L}$ rather than $7.7 \mathrm{mmol} / \mathrm{L}$ [13], but the rate of CRBSIs was relatively lower in patients with diabetes mellitus. A recent cohort study reported that relative hypoglycemia is common in ICU patients with diabetes [19].

Second, with regard to inflammatory variables, we found no statistically significant difference in the value of the index regardless of whether they were included in the variables used for adjustment. These inflammatory variables reflect the host response to "danger" in the form of infection or other insults [6], but they have poor discriminant validity due to their presence in many hospitalized patients [20].

Third, with regard to the hemodynamic variables in patients with suspected CRBSIs, the relative risk of CRBSI was significantly higher in patients with arterial hypotension $(\mathrm{MAP}<70 \mathrm{mmHg})$ than in those without hypotension, which is consistent with the SIRS criterion in patients with sepsis and can be explained by the presence of shock due to bloodstream infection, which causes circulatory abnormalities that are sufficiently profound to increase mortality [6].

Fourth, with regard to the organ dysfunction variables, the interesting finding in our study was that the rate of CRBSI was relatively lower in patients with a high creatinine level $(>133 \mu \mathrm{mol} / \mathrm{L})$, which is in contrast to the common perception and current guidelines for the care of ICU patients [12]. However, at the same time, our data show that the relative risk of CRBSI was elevated in patients receiving CRRT; CRRT is widely used in ICU patients, and although it reduces creatinine levels in patients, it does not reduce mortality [21].

Fifth, tissue perfusion variables were not helpful with regard to identifying CRBSI. Septic shock involves both hypotension and hyperlactatemia because there is not only cellular dysfunction but also cardiovascular compromise [6].

This study found that ARDS is also an independent risk factor for CRBSI. To the best of our knowledge, to date, no study has reported a direct correlation between ARDS and CRBSI. 
Table 2 Baseline cohort of patients who survived at 30 days after CVC remval due to suspected CRBSI

\begin{tabular}{|c|c|c|c|c|c|}
\hline Baseline characteristics & $\begin{array}{l}\text { All patients } \\
(n=686)\end{array}$ & $\begin{array}{l}\text { Survive } \\
(n=567)\end{array}$ & $\begin{array}{l}\text { Death } \\
(n=119)\end{array}$ & RR (95\% CI) & $\begin{array}{l}P \text { - } \\
\text { value }\end{array}$ \\
\hline Male & $503(73.3)$ & $414(73.0)$ & $89(74.8)$ & $1.079(0.740-1.574)$ & 0.6908 \\
\hline Age 1st quartile $(18-51)$ & $173(25.2)$ & $154(27.2)$ & $19(16.0)$ & $0.563(0.356-0.892)$ & 0.0106 \\
\hline Age 2nd quartile (52-66) & $174(25.4)$ & $153(27.0)$ & $21(17.6)$ & $0.631(0.407-0.978)$ & 0.0333 \\
\hline Age 3rd quartile (67-77) & $186(26.9)$ & $154(27.2)$ & $32(26.9)$ & $0.989(0.684-1.429)$ & 0.9520 \\
\hline Age 4th quartile (78-97) & $153(22.3)$ & $106(18.7)$ & $47(39.5)$ & $2.274(1.650-3.133)$ & 0.0000 \\
\hline Medical & $400(58.3)$ & $307(54.1)$ & $93(78.2)$ & $2.558(1.702-3.844)$ & 0.0000 \\
\hline Surgical & $187(27.3)$ & $172(30.3)$ & $15(12.6)$ & $0.385(0.230-0.644)$ & 0.0001 \\
\hline Traumatology & $99(14.4)$ & $88(15.5)$ & $11(9.2)$ & $0.604(0.337-1.081)$ & 0.0765 \\
\hline APACHE 1st quartile (2-15) & $195(28.4)$ & $177(31.2)$ & $18(15.1)$ & $0.449(0.280-0.720)$ & 0.0004 \\
\hline APACHE 2nd quartile (16-19) & $149(21.7)$ & $126(22.2)$ & $23(19.3)$ & $0.863(0.569-1.311)$ & 0.4863 \\
\hline APACHE 3rd quartile (20-25) & $208(30.3)$ & $174(30.7)$ & $34(28.6)$ & $0.919(0.640-1.321)$ & 0.6479 \\
\hline APACHE 4th quartile (26-46) & $134(19.5)$ & $90(15.9)$ & $44(37.0)$ & $2.417(1.754-3.331)$ & 0.0000 \\
\hline $\begin{array}{l}\text { Chronic Obstructive Pulmonary } \\
\text { Disease }\end{array}$ & $95(13.8)$ & $74(13.1)$ & $21(17.6)$ & $1.333(0.877-2.026)$ & 0.1870 \\
\hline $\begin{array}{l}\text { Acute Respiratory Distress } \\
\text { Syndrome }\end{array}$ & $76(11.1)$ & $67(11.8)$ & $9(7.6)$ & $0.657(0.348-1.241)$ & 0.1789 \\
\hline Diabetes mellitus & $121(17.6)$ & $92(16.2)$ & $29(24.4)$ & $1.505(1.040-2.177)$ & 0.0341 \\
\hline Malignancy & $73(10.6)$ & $62(10.9)$ & $11(9.2)$ & $0.855(0.483-1.514)$ & 0.5865 \\
\hline Chronic renal failure & $23(3.4)$ & $14(2.5)$ & $9(7.6)$ & $2.358(1.378-4.037)$ & 0.0050 \\
\hline Corticosteroids & $141(20.6)$ & $107(18.9)$ & $34(28.6)$ & $1.546(1.087-2.198)$ & 0.0173 \\
\hline Anticoagulant & $203(29.6)$ & $159(28.0)$ & $44(37.0)$ & $1.396(0.999-1.950)$ & 0.0523 \\
\hline $\begin{array}{l}\text { Antibiotics (Combined use [ }>2 \\
\text { types]) }\end{array}$ & $479(69.8)$ & $382(67.4)$ & $97(81.5)$ & $1.905(1.235-2.938)$ & 0.0022 \\
\hline Tracheostomy & $292(42.6)$ & $246(43.4)$ & $46(38.7)$ & $0.850(0.607-1.190)$ & 0.3427 \\
\hline Artificial respiration & $437(63.7)$ & $346(61.0)$ & $91(76.5)$ & $1.852(1.249-2.746)$ & 0.0014 \\
\hline Positive end expiratory pressure & $373(54.4)$ & $288(50.8)$ & $85(71.4)$ & $2.098(1.452-3.032)$ & 0.0000 \\
\hline CVC removal with new catheter & $484(70.8)$ & $381(67.2)$ & $103(86.6)$ & $2.687(1.629-4.432)$ & 0.0000 \\
\hline SOFA score 1st quartile $(1-8)$ & $192(28.0)$ & $174(30.7)$ & $18(15.1)$ & $0.459(0.286-0.736)$ & 0.0006 \\
\hline SOFA score 2 nd quartile $(9-10)$ & $186(27.1)$ & $161(28.4)$ & $25(21.0)$ & $0.715(0.476-1.075)$ & 0.0994 \\
\hline SOFA score 3 rd quartile (11-13) & $177(25.8)$ & $142(25.0)$ & $35(29.4)$ & $1.198(0.840-1.709)$ & 0.3222 \\
\hline SOFA score 4 th quartile (14-24) & $131(19.1)$ & $90(15.9)$ & $41(34.5)$ & $2.227(1.606-3.087)$ & 0.0000 \\
\hline CRBSI & $151(22.0)$ & $126(22.2)$ & $25(21.0)$ & $0.942(0.063-1.409)$ & 0.7714 \\
\hline Pneumonia & $412(60.1)$ & $337(59.4)$ & $75(63.0)$ & $1.134(0.807-1.592)$ & 0.4673 \\
\hline
\end{tabular}


Table 2 continued

\begin{tabular}{llllll}
\hline Baseline characteristics & $\begin{array}{l}\text { All patients } \\
(\boldsymbol{n}=\mathbf{6 8 6})\end{array}$ & $\begin{array}{l}\text { Survive } \\
(\boldsymbol{n}=\mathbf{5 6 7})\end{array}$ & $\begin{array}{l}\text { Death } \\
(\boldsymbol{n}=\mathbf{1 1 9})\end{array}$ & RR (95\% CI) & $\begin{array}{l}\boldsymbol{P} \text { - } \\
\text { value }\end{array}$ \\
\hline Abdominal Infection & $19(2.8)$ & $14(2.5)$ & $5(4.2)$ & $1.540(0.712-3.328)$ & 0.2951 \\
Gastrointestinal infection & $10(1.5)$ & $8(1.4)$ & $2(1.7)$ & $1.156(0.331-4.035)$ & 0.8234 \\
Soft tissue infections & $13(1.9)$ & $11(1.9)$ & $2(1.7)$ & $0.885(0.245-3.200)$ & 0.8504 \\
Urinary tract infections & $41(6.0)$ & $29(5.1)$ & $12(10.1)$ & $1.764(1.063-2.927)$ & 0.0376 \\
Central nervous system infections & $11(1.6)$ & $11(1.9)$ & 0 & - & 0.1256 \\
Chest infection & $1(0.1)$ & $1(0.2)$ & 0 & - & 0.6466 \\
Biliary tract infection & $2(0.3)$ & $1(0.2)$ & $1(0.8)$ & $2.900(0.718-11.701)$ & 0.2220 \\
Multiple infections & $126(18.4)$ & $102(18.0)$ & $24(20.2)$ & $1.123(0.750-1.682)$ & 0.5768 \\
Unknown & $146(21.3)$ & $124(21.9)$ & $22(18.5)$ & $0.839(0.548-1.283)$ & 0.4125 \\
\hline
\end{tabular}

Survive group and death group according to 30 days follow up

$A P A C H E$ acute physiology and chronic health evaluation, $C I$ confidence interval, CRBSI catheter-related bloodstream infection, $C V C$ central venous catheter, $I Q R$ interquartile ranges, $R R$ relative risk, $S D$ standard deviation, $S O F A$ sequential organ failure assessment, $\mathrm{PaO}_{2}$ oxygen partial pressure

Microbial pathogen-associated molecular patterns activate innate immunocytes through pattern recognition receptors, and the consequent cellular injury provides a key link between inflammation and SIRS [8]. Acute lung injury is caused by the systemic inflammatory response; dipeptidase- 1 is the target and has been shown to be a physical adhesion receptor for neutrophil sequestration independent of a major adhesion receptor on the lung [22]. Patients with CRBSI normally have severe disease, which manifests as SIRS, leading to fever and pneumonia. Hence, ARDS may be a good predictor of CRBSIs in patients with suspected CRBSIs, but the clear exclusion of other causes of lung injury is needed.

In the recent decade, these clinical data also confirmed a lower rate of CRBSI in patients with a subclavian insertion site than in those with other insertion sites [23]. Therefore, our multifactor model for the identification of CRBSI may be beneficial with regard to selecting the correct management strategy for suspected CRBSIs, and it merits further validation in a cohort study. Our results provided rational explanations for the common perceptions and current guidelines, but we do not support the use of a single risk factor as a strategy for identifying CRBSI. The complexity of real-world clinical practice is greater than that reflected in a selected cohort of patients.

When analyzing the mortality of CRBSI, which was consistent in all Cox models and subgroup analyses, CRBSIs were not associated with increased ICU mortality. The results are consistent with those of a recent cohort study that did not find differences in mortality between patients with CRBSIs and those with other ICU-acquired infections [2], another cohort study that patients with CRBSIs had lower mortality rates than those with other infections [11]. Hence, in this study, these findings regarding the relatively low mortality and attributable mortality are informative for future studies. First, most patients with suspected CRBSIs should be treated conservatively, such as with watchful waiting rather than prompt CVC removal, as is commonly performed in patients with suspected CRBSIs while waiting for blood culture results [11,12]. A recent observational study reported a treatment success rate of $85 \%$ and no deaths within six 
Table 3 The final model for time-varying analysis for the effect of CRBSI on mortality

\begin{tabular}{|c|c|c|c|c|}
\hline Sensitivity Variables & $\begin{array}{l}\text { Robust Std. } \\
\text { Err }\end{array}$ & $\begin{array}{l}z- \\
\text { Value }\end{array}$ & $\begin{array}{l}\text { Hazard ratio (95\% Confidence } \\
\text { Interval) }\end{array}$ & $\begin{array}{l}P \text { - } \\
\text { Value }\end{array}$ \\
\hline Catheter-Related Bloodstream Infection & 0.199 & -1.01 & $0.770(0.464-1.279)$ & 0.312 \\
\hline Urinary Tract Infection & 0.395 & 0.47 & $1.170(0.604-2.268)$ & 0.642 \\
\hline Age 1st quartile (18-51) (reference) & 1 & 1 & 1 & 1 \\
\hline Age 2nd quartile (52-66) & 0.318 & -0.05 & $0.982(0.521-1.851)$ & 0.956 \\
\hline Age 3rd quartile (67-77) & 0.355 & 0.41 & $1.136(0.616-2.096)$ & 0.683 \\
\hline Age 4th quartile (78-97) & 0.568 & 2.24 & $1.933(1.087-3.437)$ & 0.025 \\
\hline Medical (reference) & 1 & 1 & 1 & 1 \\
\hline Surgical & 0.114 & -3.21 & $0.398(0.227-0.698)$ & 0.001 \\
\hline Traumatology & 0.189 & -1.68 & $0.579(0.306-1.097)$ & 0.094 \\
\hline $\begin{array}{l}\text { APACHE 1st quartile }(2-15) \\
\text { (reference) }\end{array}$ & 1 & 1 & 1 & 1 \\
\hline APACHE 2nd quartile (16-19) & 0.431 & 1.01 & $1.372(0.741-2.539)$ & 0.314 \\
\hline APACHE 3rd quartile (20-25) & 0.375 & 0.67 & $1.227(0.674-2.232)$ & 0.504 \\
\hline APACHE 4th quartile (26-46) & 0.666 & 2.45 & $2.142(1.165-3.938)$ & 0.014 \\
\hline Diabetes mellitus & 0.257 & 1.08 & $1.248(0.833-1.869)$ & 0.282 \\
\hline Chronic renal failure & 0.646 & 1.34 & $1.676(0.788-3.566)$ & 0.180 \\
\hline Corticosteroids & 0.271 & 1.34 & $1.317(0.880-1.972)$ & 0.181 \\
\hline Anticoagulant & 0.169 & -1.04 & $0.803(0.532-1.214)$ & 0.299 \\
\hline Antibiotics & 0.352 & 1.33 & $1.399(0.854-2.291)$ & 0.182 \\
\hline Artificial respiration & 0.509 & 0.21 & $1.100(0.444-2.722)$ & 0.837 \\
\hline Positive end expiratory pressure & 0.761 & 1.19 & $1.702(0.708-4.088)$ & 0.234 \\
\hline Immediately new central venous catheter & 0.662 & 2.97 & $2.329(1.334-4.066)$ & 0.003 \\
\hline $\begin{array}{l}\text { SOFA score 1st quartile }(1-8) \\
\text { (reference) }\end{array}$ & 1 & 1 & 1 & 1 \\
\hline SOFA score 2nd quartile $(9-10)$ & 0.361 & 0.51 & $1.169(0.638-2.143)$ & 0.613 \\
\hline SOFA score 3rd quartile (11-13) & 0.441 & 1.3 & $1.474(0.820-2.651)$ & 0.195 \\
\hline SOFA score 4th quartile (14-24) & 0.664 & 2.65 & $2.216(1.232-3.988)$ & 0.008 \\
\hline
\end{tabular}

APACHE Acute Physiology and Chronic Health Evaluation, CRBSI: Catheter Related Bloodstream Infection, SOFA Sequential Organ Failure Assessment 


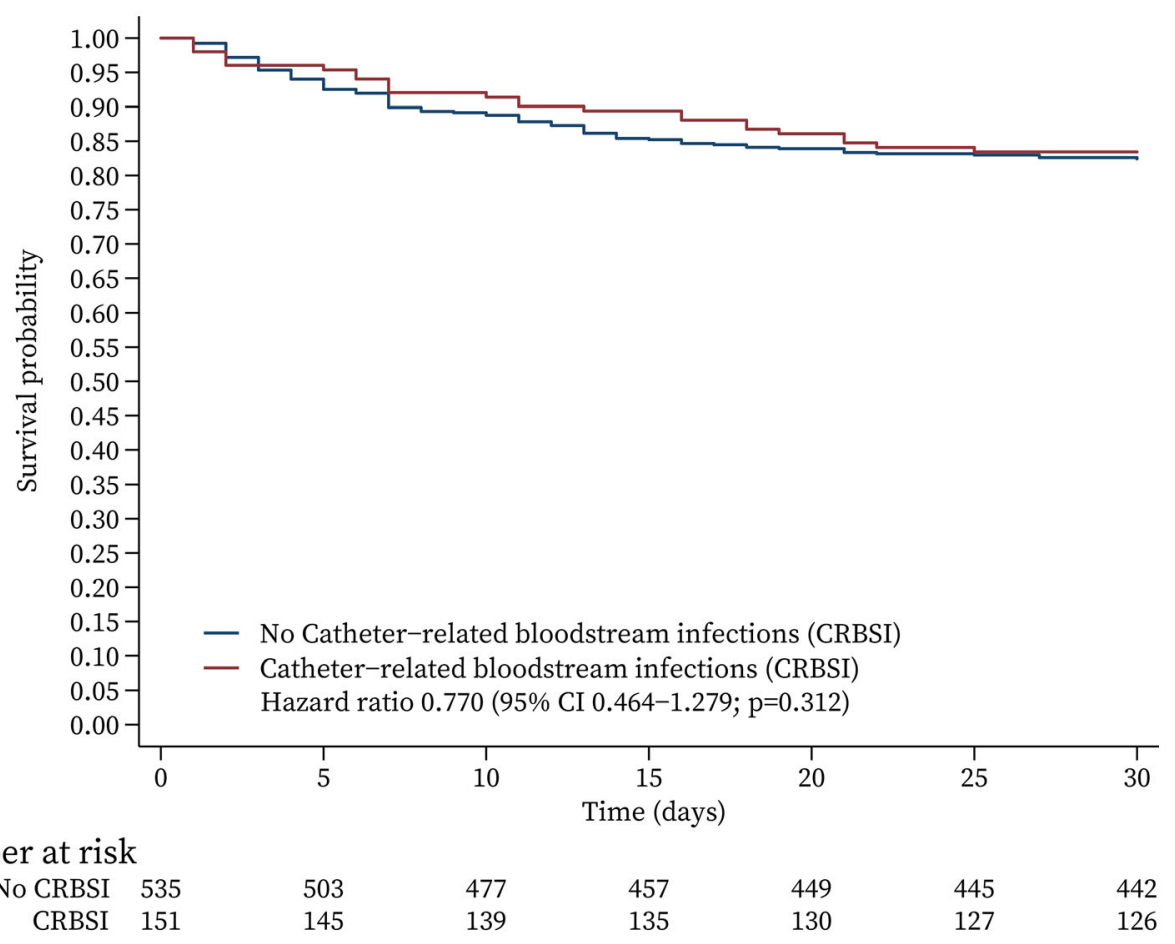

Fig. 3 Multivariate Cox survival analysis function by means of covariates. Multivariate variables including age, admission diagnosis, APACHE II score, diabetes mellitus, corticosteroids, anticoagulants, antibiotics, positive end-

weeks in hemodialysis patients with CRBSIs who did not undergo catheter removal [24]. Second, we determined that the reinsertion of a new CVC was a risk factor for mortality in our final model. Thus, a more comprehensive assessment is needed in future studies. Third, there is a lack of high-level evidence [10], and it should be possible to design a randomized controlled trial to compare mortality between patients with suspected CRBSIs managed with prompt CVC removal and those managed with watchful waiting.

The following four independent risk indicators for mortality in patients with CRBSIs were identified: age, APACHE II score, SOFA score, and reinsertion of new CVC. Other than the reinsertion of new CVCs, these risk factors for mortality have been established in previous studies [2,6]. As mentioned above, previous studies did not provide the evidence needed to assess the harm or benefit associated with this clinical practice [10], while a systematic review expiratory pressure, artificial respiration, reinsertion of new catheters, plasma procalcitonin, platelets, bilirubin, creatinine, and stratification according to mortality of CRBSI and no CRBSI

suggested that CVC removal and reinsertion may be associated with marked discomfort, severe risks and possible disruptions of or delays in treatment in critically ill patients [10]. Therefore, the potential reason underlying the increased risk of mortality in patients in whom CVCs are reinserted needs to be confirmed in future research.

The strengths of this study were that it covered the longest time span and included a list with an extensive number of known, calculable risk factors for in the analysis of attributable mortality in patients with CRBSI. However, our study has several limitations. First, this was a single-center, retrospective study that was subject to potential unmeasured confounding factors. Nevertheless, to date, it includes the largest cohort of patients with CVCs and suspected CRBSIs, and it includes more established prognostic factors than previous studies $[11,25]$, as well as known sensitive indicators for the development of CRBSI [13]. 
Second, in this cohort, it was difficult to distinguish between delayed and prompt removal [11]. However, based on the general consensus and common practice of the medical team in the participating institution, prompt CVC removal was considered a coordinate strategy in patients with suspected CRBSIs. Third, it is possible that the CVCs were not the origin of the CRBSIs in patients with suspected CRBSIs [11]. Finally, data bias could have occurred as we excluded patients without catheter tip cultures.

\section{CONCLUSION}

This initial model based on the SIRS criteria is relatively better at identifying patients with CRBSI but only in terms of the sensitivity. There were no significant differences in attributable mortality due to CRBSI and other causes in patients with suspected CRBSI, which prompt catheter removal and re-insertion of new catheter may not benefit patients with suspected CRBSIs.

\section{ACKNOWLEDGEMENTS}

The authors thank the department of medical records of Affiliated Hospital of Guangdong Medical University for clinical data support.

Funding. This study was funded by the National Natural Science Foundation of China (81671957 and 81873951) and Guangdong Natural Science Foundation (2018B030311038). In addition, data collection and storage were supported by the Clinical Research Fund of Affiliated Hospital of Guangdong Medical University (LCYJ 2018C010). The journal's Rapid Service Fee was funded by the authors.

Authorship. All named authors meet the International Committee of Medical Journal Editors (ICMJE) criteria for authorship for this article, take responsibility for the integrity of the work as a whole, and have given their approval for this version to be published.
Authorship Contributions. Yiyue Zhong and Limin Zhou contributed equally to this manuscript and Liangqing Zhang, Zhifeng Liu and Jing Tang contributed equally to this manuscript.

Disclosures. Yiyue Zhong, Limin Zhou, Xiaolei Liu, Liehua Deng, Ruona Wu, Zhengyuan Xia, Guixi Mo, Liangqing Zhang,Zhifeng Liu, Jing Tang have nothing to disclose.

Compliance with Ethics Guidelines. The ethics committee of the Affiliated Hospital of Guangdong Medical University approved this study and waived the need for informed consent on March 13, 2019 (PJ2018-066). This study was registered with the China Clinical Trials Registry on March 28, 2019 (ChiCTR1900022175). From January 2009 to December 2018.

Data Availability. The datasets generated during and/or analyzed during the current study are available from the corresponding author on reasonable request.

Open Access. This article is licensed under a Creative Commons Attribution-NonCommercial 4.0 International License, which permits any non-commercial use, sharing, adaptation, distribution and reproduction in any medium or format, as long as you give appropriate credit to the original author(s) and the source, provide a link to the Creative Commons licence, and indicate if changes were made. The images or other third party material in this article are included in the article's Creative Commons licence, unless indicated otherwise in a credit line to the material. If material is not included in the article's Creative Commons licence and your intended use is not permitted by statutory regulation or exceeds the permitted use, you will need to obtain permission directly from the copyright holder. To view a copy of this licence, visit http://creativecommons.org/licenses/by$\mathrm{nc} / 4.0 /$. 


\section{REFERENCES}

1. Takashima M, Schults J, Mihala G, Corley A, Ullman A. Complication and failures of central vascular access device in adult critical care settings. Crit Care Med. 2018;46(12):1998-2009. https://doi. org/10.1097/ccm.0000000000003370.

2. van Vught LA, Klein Klouwenberg PM, Spitoni C, et al. Incidence, risk factors, and attributable mortality of secondary infections in the intensive care unit after admission for sepsis. JAMA. 2016;315(14): 1469-79. https://doi.org/10.1001/jama.2016.2691.

3. Magill SS, Edwards JR, Bamberg W, et al. Multistate point-prevalence survey of health care-associated infections. N Engl J Med. 2014;370(13):1198-208. https://doi.org/10.1056/NEJMoa1306801.

4. Siempos II, Kopterides P, Tsangaris I, Dimopoulou I, Armaganidis AE. Impact of catheter-related bloodstream infections on the mortality of critically ill patients: a meta-analysis. Crit Care Med. 2009;37(7):2283-9. https://doi.org/10.1097/CCM. 0b013e3181a02a67.

5. Zimlichman E, Henderson D, Tamir O, et al. Health care-associated infections: a meta-analysis of costs and financial impact on the US health care system. JAMA Internal Med. 2013;173(22):2039-46. https:// doi.org/10.1001/jamainternmed.2013.9763.

6. Singer M, Deutschman CS, Seymour CW, et al. The third international consensus definitions for sepsis and septic shock (Sepsis-3). JAMA. 2016;315(8): 801-10. https://doi.org/10.1001/jama.2016.0287.

7. Weis S, Carlos AR, Moita MR, et al. Metabolic adaptation establishes disease tolerance to sepsis. Cell. 2017;169(7):1263-1275.e1214. https://doi.org/ 10.1016/j.cell.2017.05.031.

8. Zhang Q, Raoof $\mathrm{M}$, Chen $\mathrm{Y}$, et al. Circulating mitochondrial DAMPs cause inflammatory responses to injury. Nature. 2010;464(7285):104-7. https://doi.org/10.1038/nature08780.

9. Ziegler MJ, Pellegrini DC, Safdar N. Attributable mortality of central line associated bloodstream infection: systematic review and metaanalysis. Infection. 2015;43(1):29-36. https://doi. org/10.1007/s15010-014-0689-y.

10. Janum S, Afshari A. Central venous catheter (CVC) removal for patients of all ages with candidaemia. Cochrane Database Syst Rev. 2016;7(7):Cd011195. doi:https://doi.org/10.1002/14651858.CD011195. pub2

11. Lorente L, Martin MM, Vidal P, Rebollo S, Ostabal MI, Sole-Violan J. Should central venous catheter be systematically removed in patients with suspected catheter related infection? Crit Care. 2014;18(5): 564. https://doi.org/10.1186/s13054-014-0564-3.

12. Mermel LA, Allon M, Bouza E, et al. Clinical practice guidelines for the diagnosis and management of intravascular catheter-related infection: 2009 Update by the Infectious Diseases Society of America. Clin Infect Dis. 2009;49(1):1-45. https://doi. org/10.1086/599376.

13. Levy MM, Fink MP, Marshall JC, et al. 2001 SCCM/ ESICM/ACCP/ATS/SIS international sepsis definitions conference. Crit Care Med. 2003;31(4): 1250-6. https://doi.org/10.1097/01.CCM. 0000050454.01978.3B.

14. Dellinger RP, Levy MM, Rhodes A, et al. Surviving Sepsis Campaign: international guidelines for management of severe sepsis and septic shock, 2012. Intensive Care Med. 2013;39(2):165-228. https://doi.org/10.1007/s00134-012-2769-8.

15. Soufir L, Timsit JF, Mahe C, Carlet J, Regnier B, Chevret S. Attributable morbidity and mortality of catheter-related septicemia in critically ill patients: a matched, risk-adjusted, cohort study. Infect Control Hosp Epidemiol. 1999;20(6):396-401. https://doi.org/10.1086/501639.

16. Rawshani A, Rawshani A, Franzén S, et al. Risk factors, mortality, and cardiovascular outcomes in patients with type 2 diabetes. $\mathrm{N}$ Engl J Med. 2018;379(7):633-44. https://doi.org/10.1056/ NEJMoa1800256.

17. Xue X, Xie X, Gunter M, et al. Testing the proportional hazards assumption in case-cohort analysis. BMC Med Res Methodol. 2013;13:88. https://doi. org/10.1186/1471-2288-13-88.

18. Tijmstra J. Why checking model assumptions using null hypothesis significance tests does not suffice: A plea for plausibility. Psychon Bull Rev. 2018;25(2): 548-59. https://doi.org/10.3758/s13423-018-14474.

19. Kwan TN, Zwakman-Hessels L, Marhoon N, et al. Relative hypoglycemia in diabetic patients with critical illness. Crit Care Med. 2019;48(3):e233-40. https://doi.org/10.1097/ccm.0000000000004213.

20. Churpek MM, Zadravecz FJ, Winslow C, Howell $\mathrm{MD}$, Edelson DP. Incidence and prognostic value of the systemic inflammatory response syndrome and organ dysfunctions in ward patients. Am J Respir Crit Care Med. 2015;192(8):958-64. https://doi.org/ 10.1164/rccm.201502-0275OC.

21. Gaudry S, Hajage D, Schortgen F, et al. Initiation strategies for renal-replacement therapy in the 
intensive care unit. N Engl J Med. 2016;375(2): 122-33. https://doi.org/10.1056/NEJMoa1603017.

22. Choudhury SR, Babes L, Rahn JJ, et al. Dipeptidase1 is an adhesion receptor for neutrophil recruitment in lungs and liver. Cell. 2019;178(5):12051221.e1217. https://doi.org/10.1016/j.cell.2019.07. 017.

23. Parienti JJ, Mongardon N, Megarbane B, et al. Intravascular complications of central venous catheterization by insertion site. $\mathrm{N}$ Engl J Med. 2015;373(13):1220-9. https://doi.org/10.1056/ NEJMoa1500964.
24. Mandolfo S, Anesi A, Maggio M, Rognoni V, Galli F, Forneris G. High success rate in salvage of catheterrelated bloodstream infections due to Staphylococcus aureus, on behalf of project group of Italian society of nephrology. J Vasc Access. 2020;21(3): 336-41. 1129729819875323.

25. Mishra SB, Misra R, Azim A, et al. Incidence, risk factors and associated mortality of central line-associated bloodstream infections at an intensive care unit in northern India. Int J Quality Health Care. 2017;29(1):63-7. https://doi.org/10.1093/intqhc/ mzw144. 